\title{
Economic Dispatch Solution Using Moth-Flame Optimization Algorithm
}

\author{
Mohd Herwan Sulaiman ${ }^{1}$, Zuriani Mustaffa ${ }^{2}$, Muhammad Ikram Mohd Rashid ${ }^{1}$ and Hamdan Daniyal ${ }^{1}$ \\ ${ }^{1}$ Faculty of Electrical \& Electronics Engineering, Universiti Malaysia Pahang, 26600 Pekan Pahang, Malaysia \\ ${ }^{2}$ Faculty of Computer Systems and Software Engineering, Universiti Malaysia Pahang, 26300Gambang Pahang, Malaysia
}

\begin{abstract}
This paper proposes an application of a recent nature inspired optimization technique namely Moth-Flame Optimization (MFO) algorithm in solving the Economic Dispatch (ED) problem. In this paper, the practical constraints will be included in determining the minimum cost of power generation such as ramp rate limits, prohibited operating zones and generators operating limits. To show the effectiveness of proposed algorithm, two case systems are used: 6-units and 15-units systems and then the performance of MFO is compared with other techniques from literature. The results show that MFO is able to obtain less total cost than those other algorithms.
\end{abstract}

\section{Introduction}

Economic Dispatch (ED) is one of the complex problems in power system operation that draws a lot of attention of many researchers. The task of ED is to find the optimal power generation to meet the demand at the minimum cost by fulfilling all the constraints. In addition, by taking the practical and operational constraints into account, it makes ED highly nonlinear constrained optimization problem, especially for larger systems. Any improvements in optimal output scheduling can contribute significantly in terms of cost savings for a long run.

To date, there are massive techniques and algorithms that have been applied to solve the ED problems. The application of nature inspired algorithms becoming the popular choices of many researchers that have been proven through literatures. The implementation of Particle Swarm Optimization (PSO) into ED solution has been proposed in [1]. The variants of Genetic Algorithm (GA) namely Beeder GA (BGA), Fast Navigating GA (FNGA), Twin Removal GA (TRGA), Kite GA (KGA) and United GA 9UGA) have been proposed in [2] to solve ED problems. Nevertheless, the results presented in [2] still unsatisfactory if compared with the Backtracking Search Algorithm that has been proposed in [3].

Ref. [4] has proposed Cuckoo Search Algorithm (CSA) and the Firefly Algorithm (FA) has been proposed in [5] to solve ED problems. The modification of FA (MFA) based on [5] has been proposed in [6] to solve ED problems with practical constraints. Other algorithms that also have been proposed in literature to solve ED are such as Grey Wolf Optimizer (GWO) [7], Flower Pollination Algorithm (FPA) [8], Whale Optimization Algorithm
(WOA) [9], Improve/ modified PSO [10, 11] and Hybrid Bee Algorithm with Tabu Search (BA/TS) [12].

In this paper, the recent developed algorithm namely Moth-Flame Optimization (MFO) algorithm has been applied to solve practical ED problems. MFO is inspired by moth's navigation at night proposed by [13]. The rest of the paper is organized as follows. Section 2 discuss the ED problem formulation followed by the MFO algorithm in Section 3. Section 4 presents the implementation of MFO in solving ED problem and Section 5 presents the results and discussion. Finally, the conclusion is stated in Section 6 .

\section{Economic Dispatch}

Economic Dispatch (ED) is about to find the optimal power generation at the lowest cost to reliably serve the loads subject to the constraints. In ED, the cost function can be mathematical described as the quadratic function, as follows:

$$
F_{i}\left(P_{i}\right)=\alpha_{i} P_{i}^{2}+\beta_{i} P_{i}+\gamma_{i}
$$

where $\alpha_{i}, \beta_{i}$ and $\gamma_{i}$ are the coefficients of generator $i$. The objective function to be minimized are the summation of cost function in (1), which can be expressed as follows:

$$
\min f=\sum_{i=1}^{n} F_{i}\left(P_{i}\right)
$$

where $n$ is the number of generator units. Eqns. (1) and (2) are subject to the power balanced equation as equality constraint to be satisfied where can be expressed as follows: 


$$
\sum_{i=1}^{n}\left(P_{i}\right)=P_{\text {Demand }}+P_{\text {Loss }}
$$

where $P_{\text {Demand }}$ is the total load demand, $P_{\text {Loss }}$ is the total loss of the power system. Since the losses in the interconnected power system cannot be avoided, the $B$ coefficient method is used to calculate the losses apart of load flow calculation, as follows:

$$
P_{\text {Loss }}=\sum_{i=1}^{n} \sum_{j=1}^{n} P_{i} B_{i j} P_{j}+\sum_{i=1}^{n} B_{0 i} P_{i}+B_{00}
$$

For the inequality constraint, the power generation must be operated within its individual limits, as follows:

$$
P_{i(\min )} \leq P_{i} \leq P_{i(\max )} \quad i=1,2, \ldots, n
$$

Other than that, by considering the practical constraints, the operating range of all generation units are restricted by their ramp rate limits [14], which are expressed as follow:

$$
\begin{aligned}
& P_{i}-P_{i}^{o} \leq U R_{i} \quad \text { if generation is increased } \\
& P_{i}^{o}-P_{i} \leq D R_{i} \quad \text { if generation is decreased }
\end{aligned}
$$

where $P_{i}^{o}$ is the previous power generation of unit $i$. $U R_{i}$ and $D R_{i}$ are the up-ramp and down-ramp limits in $\mathrm{MW} / \mathrm{h}$ of the $i$-th generator, respectively. The generator operation constraints with the ramp rate limit now becomes as follows:

$\max \left(P_{i}^{\min }, U R_{i}-P_{i}^{o}\right) \leq P_{i} \leq \min \left(P_{i}^{\max }, P_{i}^{o}-D R_{i}\right)$

The other practical constraint that will be considered in this paper is the prohibited operating zones for the generators. The condition of this situation is visually shown in Fig. 1. Since the shape of the input-output curve in the neighborhood of the prohibited zones are difficult to determined, the best economical way is by avoiding the operation in these areas. The feasible operating zones of a unit can be determined as follow [15]:

$$
\left\{\begin{array}{l}
P_{i}^{\min } \leq P_{i} \leq P_{i, 1}^{\text {lower }} \\
P_{i, j-1}^{\text {upper }} \leq P_{i} \leq P_{i, j}^{\text {lower }} \\
P_{i, P Z_{i}}^{\text {upper }} \leq P_{i} \leq P_{i}^{\max }
\end{array}, j=2,3, \ldots, P Z_{i}\right.
$$

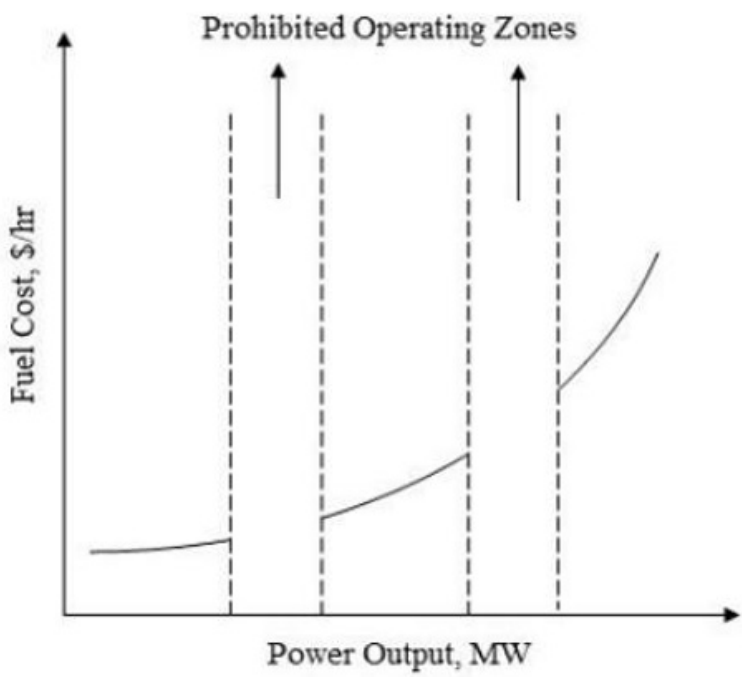

Figure 1. Fuel cost curve with prohibited zone

\section{Moth-Flame Optimization Algorithm}

MFO algorithm is inspired by the moth's special navigation at night proposed by [13]. Moths fly at night by referring to the moon light as orientation for navigation. They fly by maintaining a fixed angle with respect to the moon for straight path. Moths are tricked by the artificial light which make them fly spirally around the lights. However, the effort of moths to maintain a similar angle to the light source which is extremely close compared to the moon will causes a deadly spiral fly path for them. This behavior is depicted in the Fig. 2.

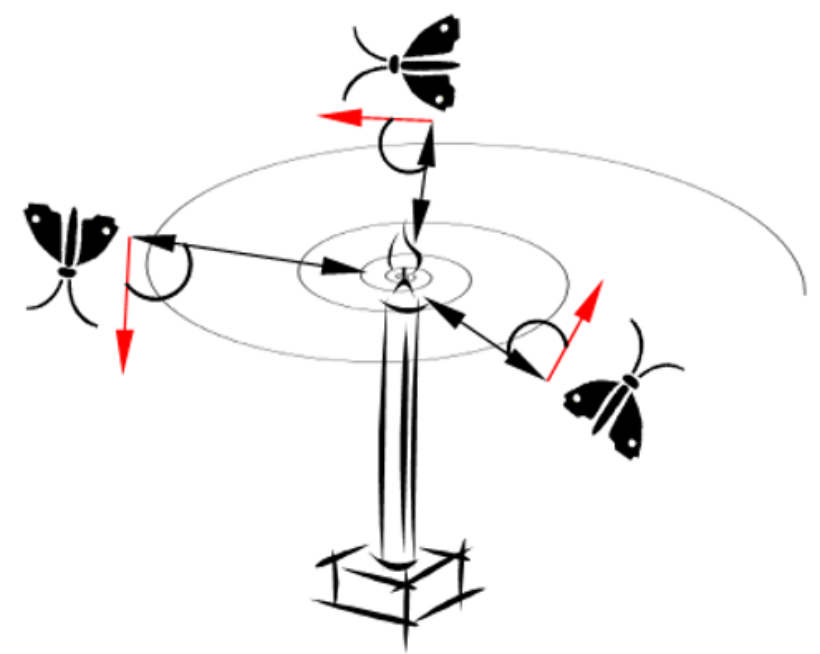

Figure 2. Spiral flying path around close light sources [13].

The development of MFO initially can be described as the following expression:

$$
M=\left[\begin{array}{cccc}
m_{1,1} & m_{1,2} & \cdots & m_{1, d} \\
\vdots & \vdots & \cdots & \vdots \\
\vdots & \vdots & \cdots & \vdots \\
m_{n, 1} & m_{n, 2} & \cdots & m_{n, d}
\end{array}\right]
$$


where $M$ is a set of moths, $n$ is the number of moths and $d$ is the number of variables (dimension). It is assumed that the candidate of solutions is moths. Other key components in MFO of course are the flames which are constituted also in the form of matrix as follows:

$$
F=\left[\begin{array}{cccc}
F_{1,1} & F_{1,2} & \cdots & F_{1, d} \\
\vdots & \vdots & \cdots & \vdots \\
\vdots & \vdots & \cdots & \vdots \\
F_{n, 1} & F_{n, 2} & \cdots & F_{n, d}
\end{array}\right]
$$

where $n$ and $d$ are the number of moth and dimension respectively. Both (10) and (11) are assumed to store the corresponding fitness values in an array as expressed below:

$$
\begin{gathered}
O M=\left[\begin{array}{c}
O M_{1} \\
\vdots \\
\vdots \\
O M_{n}
\end{array}\right] \\
O F=\left[\begin{array}{c}
O F_{1} \\
\vdots \\
\vdots \\
O F_{n}
\end{array}\right]
\end{gathered}
$$

From (12) and (13), both moth and flame are solutions. The difference between them is how to treat and update them for each iteration. In MFO, the moths are actual search agents that move around the search space, whereas flames are the best position of moths so far. Thus, flames can be treated as flags that are dropped by moths when searching the search space. In this mechanism, a moth never loses its best solution [13].

The position of each moth is updated with respect to the flame by using the following expression:

$$
M_{i}=S\left(M_{i}, F_{j}\right)
$$

where $M_{i}$ indicates the $i$-th moth, $F_{j}$ is the $j$-th flame and $S$ is the spiral function. A logarithmic spiral function is selected as the main update mechanism of moth, such as [13]:

$$
S\left(M_{i}, F_{j}\right)=D_{i} \cdot e^{b t} \cdot \cos (2 \pi t)+F_{j}
$$

where $D_{i}$ is the distance of the $i$-th moth for the $j$-th flame, $b$ is a constant for defining the shape of the logarithmic spiral and $t$ is a random number between -1 and 1 . To prevent from trapped in local optima, each moth is obliged to update its position using one flame only in eqn. (15). At each iteration and after updating the flames, the flames are sorted based on the fitness values. Then the moths are updating their position with respect to the corresponding flames. Details description of MFO can be obtained in [13].

\section{MFO for ED Problem}

The implementation of MFO in solving the ED problem is by obtaining the optimal values power generation while satisfying all the constrained mentioned in section 2 . Initially, the number of moths or search agents and maximum iteration are set. The population (candidate for solution) is constructed in matrix form as depicted in eqn. (10) where the row represents the number of moths and the column represents the number of control variables to be optimized (power generation).

To obtain the objective function, each position of moth is evaluated to obtain the total cost using (2). Once the total cost has obtained for respected moth (after updating with the flames such in eqn. (11-14)), the matrix is sorted where the best solution so far is located at the top while the worst result is located at the bottom of the population matrix. If the updated variables are out of bound from the constraints, they are pegged at the minimum or maximum boundaries so that the result obtained is correct. To deal with the equality constraint such in (3), the penalty method was used. The penalty is reflected to the power balance mismatch and embedded in the cost function (2) as follows:

$$
F=(F)+P F * a b s\left[\left(\sum_{i=1}^{n} P_{i}\right)-P_{\text {Demand }}-P_{\text {Loss }}\right]
$$

where $P F$ is the penalty factor. The implementation of MFO in solving ORPD is exhibited in Fig. 3.

\section{Results and Discussion}

To show the effectiveness of proposed MFO in solving ED problem, 2 test systems are used: 6-units and 15-units generation systems. The simulation was implemented in MATLAB. 


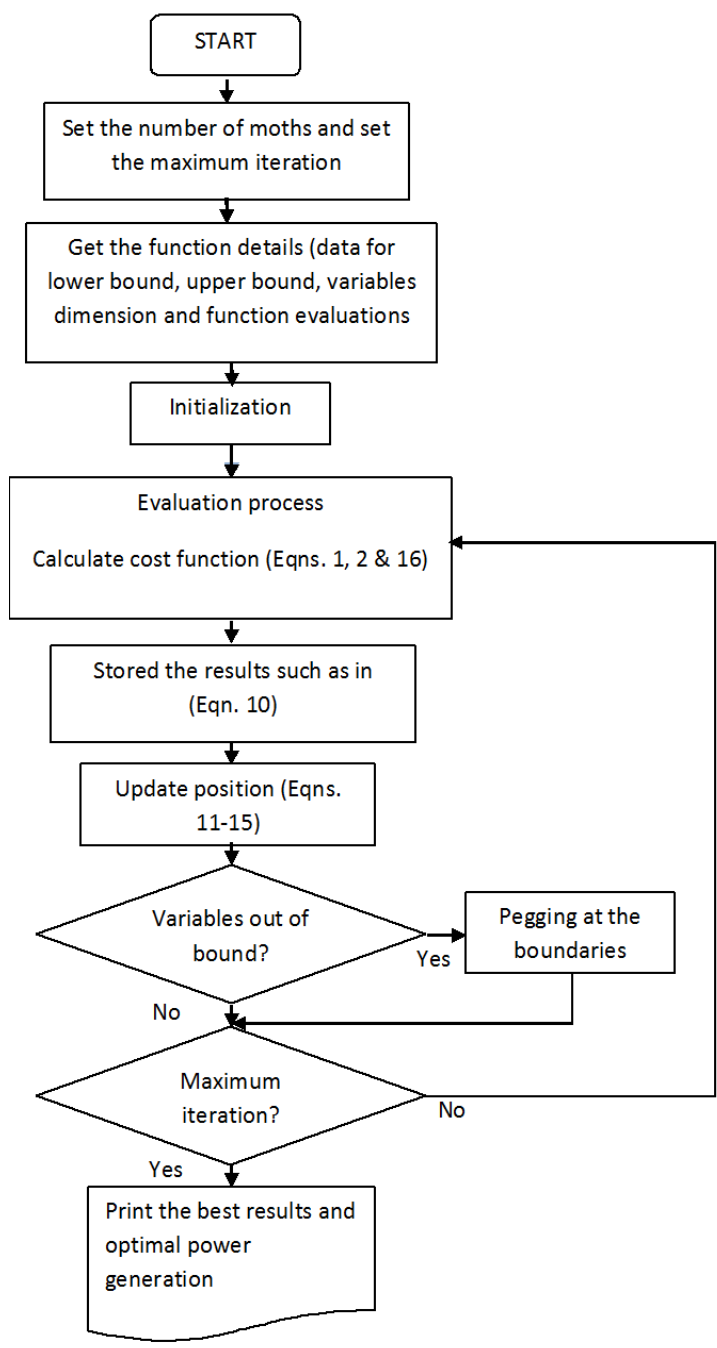

Figure 3. Flow of proposed MFO for solving ED problem

\subsection{6-units system}

The system consists of 6 thermal units in 26 bus system including 46 transmission lines. The load demand is set to $1263 \mathrm{MW}$. The characteristics of this system can be obtained in [6] and the B-loss coefficients can be obtained in [1].

The best results of MFO with other methods such as Kite Genetic Algorithm (KGA) [2], PSO [1], Backtracking Search Algorithm (BSA) [3] and Modified Firefly Algorithm (MFA) [6] are tabulated in Table I. From this table, it can be seen that the proposed MFO has a better solution in terms of total cost generated compared to others. MFO also gave the best results in terms of minimum line loss which is $12.414 \mathrm{MW}$.
Table 1. Best ED Solution for 6-units system

\begin{tabular}{|l|l|l|l|l|l|}
\hline $\begin{array}{l}\text { Generator } \\
\text { Output }\end{array}$ & KGA & PSO & BSA & MFA & MFO \\
\hline P1 & 447.66 & 447.5 & 447.4902 & 445.08 & 446.71 \\
\hline P2 & 173.60 & 173.32 & 173.3308 & 173.08 & 173.22 \\
\hline P3 & 262.91 & 263.47 & 263.4559 & 264.42 & 263.96 \\
\hline P4 & 139.18 & 139.06 & 139.0602 & 139.59 & 139.2 \\
\hline P5 & 165.58 & 165.48 & 165.4804 & 166.02 & 165.68 \\
\hline P6 & 87.03 & 87.13 & 87.1409 & 87.21 & 86.64 \\
\hline Line Loss & 12.96 & 12.958 & 12.9583 & 12.41 & 12.414 \\
\hline Cost $(\$ / h)$ & 15449.89 & 15,450 & $15,449.89$ & 15,443 & 15442.66 \\
\hline
\end{tabular}

To analyze the performance of MFO in optimizing the power generation for this system, various number of search agents are used: 20, 30 and 40. Fig. 4 shows the performance in terms of total generation cost $(\$ / \mathrm{hr})$ versus free runs for various numbers of search agents of MFO. It can be noted that the results of 20 search agents are slightly better compared to others in obtaining the minimum cost which is at the run \# 24 which is the best cost of $\$ 15,442.66 / \mathrm{hr}$. However, 30 and 40 search agents are quite stable and consistent compared to 20 search agents' solutions. Fig. 5 shows the convergence curve of the best results of each number of search agents among 30 free running simulations. It can be noted that all search agents are converged less than 70 iterations.

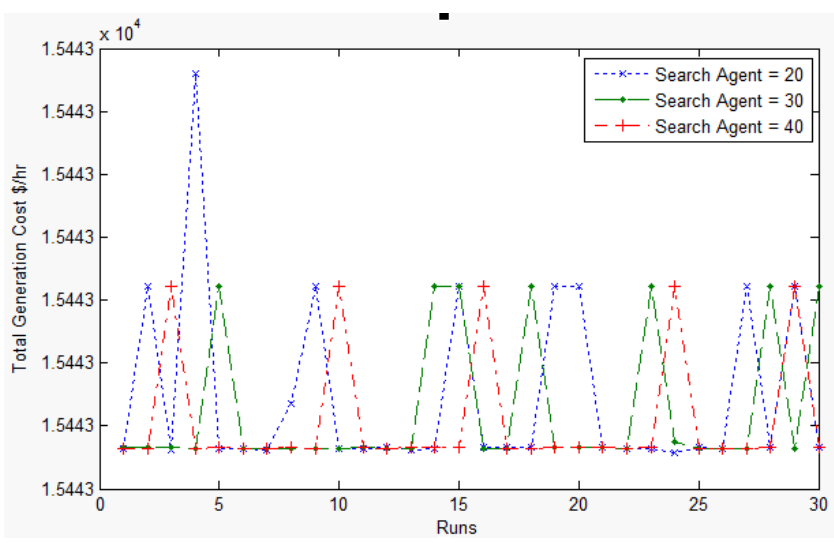

Figure 4. Performance for 20, 30 and 40 search agents for 30 free running simulations for 6 -units system.

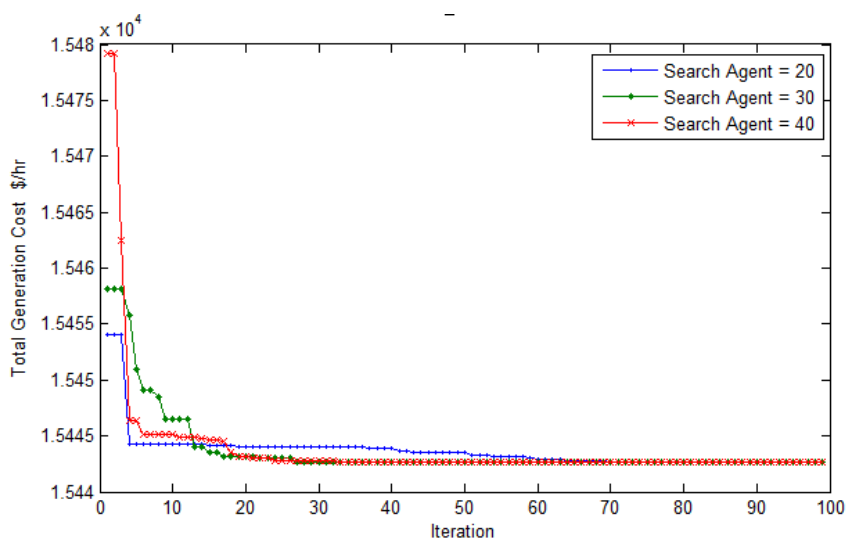

Figure 5. Convergence curve of 20, 30 and 40 search agents for 6-units system. 


\subsection{5 -units system}

For this system, the load demand is set to 2630. The generators' characteristics can be obtained in [1]. The prohibited operating zones embedded in the units 2, 5, 6 and 12. A detailed results of the optimal ED solution for this system of MFO together with Twin Removal Genetic Algorithm (TRGA) [2], BSA [3], FA [5] and Cuckoo Search Algorithm (CSA) [4] are tabulated in Table II. It can be noted that MFO gave the best results in terms of minimum cost of generation: $\$ 32,687.02 / \mathrm{hr}$ which is less $\$ 0.02 / \mathrm{hr}$ compared to CSA [4]. However, the total loss obtained by MFO is better where MFO produces $30.0522 \mathrm{MW}$ compared to CSA, which is $30.0541 \mathrm{MW}$.

Again, in order to analyze the performance of MFO in optimizing the power generation for this system, 20, 30 and 40 search agents are used. Fig. 6 shows the convergence curve of the best results of each number of search agents among 30 free running simulations. It can be concluded that all search agents are converged less than 100 iterations. Fig. 7 shows the performance in terms of total generation cost $(\$ / \mathrm{hr})$ versus free runs for various numbers of search agents of MFO. It can be seen that the results of 30 search agents are slightly better compared to others in obtaining the minimum cost which is at the run \# 17 which is the best cost of $\$ 32,687.02 / \mathrm{hr}$. Nevertheless, 40 search agents gave the consistent results throughout 30 free running simulations.

TABLE 2. Best ED SOLUTION For 15-UNITS SYSTEM

\begin{tabular}{|l|l|l|l|l|l|}
\hline \multirow{2}{*}{ Unit } & TRGA & BSA & FA & CSA & MFO \\
\cline { 2 - 6 } & $(\mathrm{MW})$ & $(\mathrm{MW})$ & $\mathrm{MW})$ & $(\mathrm{MW})$ & $(\mathrm{MW})$ \\
\hline 1 & 455.00 & 455.00 & 454.97 & 455.00 & 455.00 \\
\hline 2 & 380.00 & 380.00 & 380.00 & 380.00 & 380.00 \\
\hline 3 & 130.00 & 130.00 & 130.00 & 130.00 & 130.00 \\
\hline 4 & 130.00 & 130.00 & 130.00 & 130.00 & 130.00 \\
\hline 5 & 169.97 & 170.00 & 169.80 & 170.00 & 170.00 \\
\hline 6 & 460.00 & 460.00 & 460.00 & 460.00 & 460.00 \\
\hline 7 & 430.00 & 430.00 & 430.00 & 430.00 & 430.00 \\
\hline 8 & 69.38 & 71.64 & 86.89 & 84.94 & 80.78 \\
\hline 9 & 61.30 & 59.02 & 47.26 & 44.22 & 48.36 \\
\hline 10 & 159.97 & 160.00 & 155.20 & 160.00 & 160.00 \\
\hline 11 & 80.00 & 80.00 & 79.87 & 80.00 & 80.00 \\
\hline 12 & 80.00 & 80.00 & 79.93 & 80.00 & 80.00 \\
\hline 13 & 25.03 & 25.00 & 25.00 & 25.00 & 25.00 \\
\hline 14 & 15.00 & 15.00 & 15.05 & 15.00 & 15.00 \\
\hline 15 & 15.00 & 15.00 & 15.22 & 15.00 & 15.00 \\
\hline $\begin{array}{l}\text { Loss } \\
(\mathrm{MW})\end{array}$ & 30.65153 & 30.6609 & 30.1329 & 30.0541 & $\mathbf{3 0 . 0 5 2 2}$ \\
\hline $\begin{array}{l}\text { Cost } \\
(\$ \mathbf{h})\end{array}$ & $32,704.53$ & $32,704.45$ & $32,689.39$ & 32687.04 & $\mathbf{3 2 , 6 8 7 . 0 2}$ \\
\hline
\end{tabular}

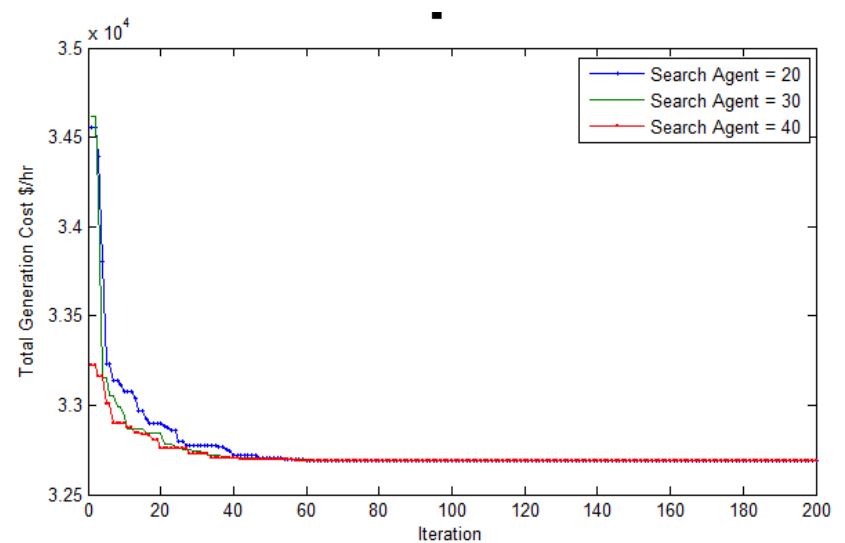

Figure 6.Convergence curve of 20,30 and 40 search agents for 6-units system.

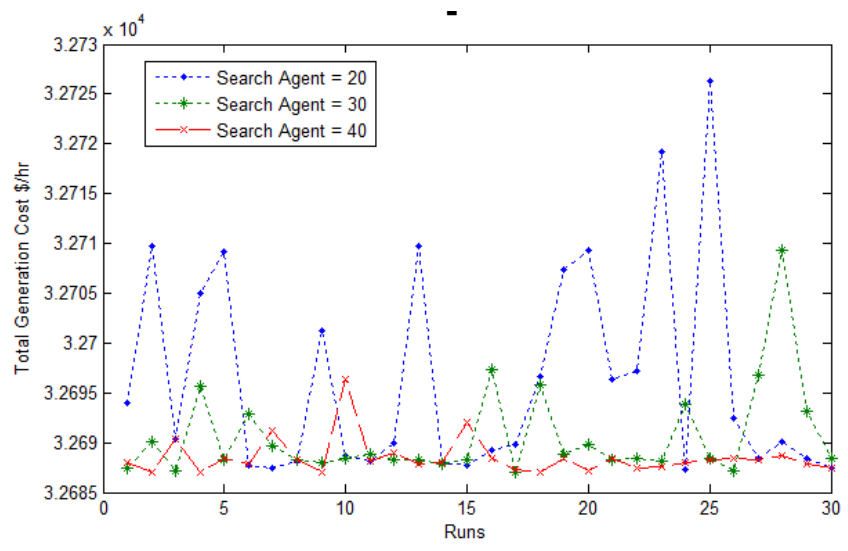

Figure 7.Performance for 20, 30 and 40 search agents for 30 free running simulations for 15 -units system.

\section{Conclusion}

This paper has proposed a recent nature inspired computing algorithm, Moth-Flame Optimization algorithm in solving economic dispatch problem. The effectiveness of MFO was demonstrated using 6-units and 15-units systems. Simulation results showed that MFO is better compared to other selected algorithms in terms of finding the minimum cost of generation in $\$ / \mathrm{hr}$. The implementation of MFO into economic emission dispatch will be proposed in the near future.

\section{Acknowledgment}

This research study is supported by Ministry of Higher Education Malaysia (MoHE) and Universiti Malaysia Pahang under Fundamental Research Grant Scheme \#RDU 170129.

\section{References}

1. G. Zwe-Lee, "Particle swarm optimization to solving the economic dispatch considering the generator constraints," IEEE Transactions on Power Systems, vol. 18, pp. 1187-1195, 2003.

2. S. Rastgoufard, S. Iqbal, M. T. Hoque, and D. Charalampidis, "Genetic algorithm variant based effective solutions for economic dispatch problems," in 2018 IEEE Texas Power and Energy Conference (TPEC), 2018, pp. 1-6. 
3. M. Modiri-Delshad, S. H. Aghay Kaboli, E. Taslimi-Renani, and N. A. Rahim, "Backtracking search algorithm for solving economic dispatch problems with valve-point effects and multiple fuel options," Energy, vol. 116, pp. 637-649, 2016/12/01/ 2016.

4. M. H. Sulaiman and M. R. Mohamed, "Solving economic dispatch problems utilizing Cuckoo Search algorithm," in 2014 IEEE 8th International Power Engineering and Optimization Conference (PEOCO2014), 2014, pp. 89-93.

5. X.-S. Yang, S. S. Sadat Hosseini, and A. H. Gandomi, "Firefly Algorithm for solving non-convex economic dispatch problems with valve loading effect," Applied Soft Computing, vol. 12, pp. 1180-1186, 2012/03/01/ 2012

6. M. H. Sulaiman, H. Daniyal, and M. W. Mustafa, "Modified Firefly Algorithm in solving economic dispatch problems with practical constraints," in 2012 IEEE International Conference on Power and Energy (PECon), 2012, pp. 157-161.

7. L. I. Wong, M. H. Sulaiman, M. R. Mohamed, and M. S. Hong, "Grey Wolf Optimizer for solving economic dispatch problems," in 2014 IEEE International Conference on Power and Energy (PECon), 2014, pp. 150-154.

8. S. Vijayaraj and R. K. Santhi, "Multi-area economic dispatch using flower pollination algorithm," in 2016 International Conference on Electrical, Electronics, and Optimization Techniques (ICEEOT), 2016, pp. 4355-4360.

9. F. Mohamed, M. Abdel-Nasser, K. Mahmoud, and S. Kamel, "Economic dispatch using stochastic whale optimization algorithm," in 2018 International Conference on Innovative Trends in Computer Engineering (ITCE), 2018, pp. 19-24.

10. H. Lotfi, A. Dadpour, and M. Samadi, "Solving economic dispatch in competitive power market using improved particle swarm optimization algorithm," in 2017 Conference on Electrical Power Distribution Networks Conference (EPDC), 2017, pp. 188-195.

11. K. Zare and T. G. Bolandi, "Modified iteration particle swarm optimization procedure for economic dispatch solving with nonsmooth and non-convex fuel cost function," in $3 r d$ IET International Conference on Clean Energy and Technology (CEAT) 2014, 2014, pp. 1-6

12. S. Chansareewittaya, "Hybrid BA/TS for economic dispatch considering the generator constraint," in 2017 International Conference on Digital Arts, Media and Technology (ICDAMT), 2017, pp. 115-119.

13. S. Mirjalili, "Moth-flame optimization algorithm: A novel natureinspired heuristic paradigm," Knowledge-Based Systems, vol. 89, pp. 228-249, 2015/11/01/2015.

14. C. Po-Hung and C. Hong-Chan, "Large-scale economic dispatch by genetic algorithm," IEEE Transactions on Power Systems, vol. 10, pp. 1919-1926, 1995.

15. C.-C. Kuo, "A novel string structure for economic dispatch problems with practical constraints," Energy Conversion and Management, vol. 49, pp. 3571-3577, 2008/12/01/2008 